\title{
On Approximate Solutions of Systems of Linear Inequalities*
}

\begin{abstract}
Alan J. Hoffman
Let $A \boldsymbol{x} \leqq \boldsymbol{b}$ be a consistent system of linear inequalities. The principal result is a quantitative formulation of the fact that if $x$ "almost" satisfies the inequalities, then $x$ is "close" to a solution. It is further shown how it is possible in certain cases to estimate the size of the vector joining $x$ to the nearest solution from the magnitude of the positive coordinates of $\boldsymbol{A x}-\boldsymbol{b}$.
\end{abstract}

\section{Introduction}

In many computational schemes for solving a system of linear inequalities

$$
\begin{gathered}
A_{1} \cdot \boldsymbol{x}=a_{11} x_{1}+\ldots+a_{1 n} x_{n} \leqq b_{1} \\
\cdot \\
\cdot \\
\mathrm{A}_{m} \cdot \boldsymbol{x}=a_{m 1} x_{1}+\ldots+a_{m n} x_{n} \leqq b_{m}
\end{gathered}
$$

(briefly, $A \boldsymbol{x} \leqq \boldsymbol{b}$ ), one arrives at a vector $\overline{\boldsymbol{x}}$ that "almost" satisfies (1). It is almost obvious geometrically that, if (1) is consistent, one can infer that there is a solution $\boldsymbol{x}_{0}$ of (1) "close" to $\overline{\boldsymbol{x}}$. The purpose of this report is to justify and formulate precisely this assertion. ${ }^{1}$ We shall use fairly general definitions of functions that measure the size of vectors, since it may be possible to obtain better estimates of the constant $c$ (whose important role is described in the statement of the theorem) for some measuring functions than for others. We shall make a few remarks on the estimation of $c$ after completing the proof of the main theorem.

\section{The Main Theorem}

We require the following Definitions:

For any real number $a$, we define

$$
a^{+}={ }_{0}^{a} \text { if } a<0 .
$$

For any vector $\boldsymbol{y}=\left(y_{1}, \ldots, y_{k}\right)$, we define

$$
\boldsymbol{y}^{+}=\left(y_{1}^{+}, . ., y_{k}^{+}\right) \text {. }
$$

A positive homogeneous function $F_{k}$ defined on $k$-space is a real continuous function satisfying

(i) $\quad F_{k}(\boldsymbol{x}) \geqq 0, \quad F_{k}(\boldsymbol{x})=0$ if, and only if, $\boldsymbol{x}=\mathbf{0}$

$$
\text { (ii) } \quad \alpha \geqq 0 \text { implies } F_{k}(\alpha \boldsymbol{x})=\alpha F_{k}(\boldsymbol{x})
$$

*This work was sponsored (in part) by the Office of Scientific Research, USA F. ${ }_{1}$ A. M. Ostrowski has kindly pointed out that part of the results given below is implied by the fact that if $K$ and $L$ are two convex bodies each of which is in a small neighborhood of the other, then their associated gauge functions differ slightly.
Theorem: Let (1) be a consistent system of inequalities and let $F_{n}$ and $F_{m}$ each satisfy (3). Then there exists a constant $c>0$ such that for any $\boldsymbol{x}$ there exists a solution $\boldsymbol{x}_{0}$ of (1) with

$$
\left.F_{n}\left(\boldsymbol{x}-\boldsymbol{x}_{0}\right) \leqq c F_{m}(A \boldsymbol{x}-b)^{+}\right)
$$

The proof is essentially contained in two lemmas ( 2 and 3 below) given by Shmuel Agmon. ${ }^{2}$

Lemma 1. If $F_{m}$ satisfies (3), there exists an $e>0$ such that for every $\boldsymbol{y}$ and every subset $S$ of the half spaces (1)

$$
F_{m}(\overline{\boldsymbol{y}}) \leqq e F_{m}(\boldsymbol{y})
$$

where $\boldsymbol{y}=\left(y_{1}, \ldots, y_{m}\right), \overline{\boldsymbol{y}}=\left(\bar{y}_{1}, \ldots, \bar{y}_{m}\right)$, and

$$
\begin{aligned}
& \bar{y}_{i}=y_{i} \text { if the } i \text { th half space belongs to } S \\
& 0 \quad \text { otherwise. }
\end{aligned}
$$

Proof. It is clear from (3) (i) that any $e$ will suffice for $y=0$. By (3) (ii), we need only consider the ratio $F_{m}(\overline{\boldsymbol{y}}) / F_{m}(\boldsymbol{y})$ for $\boldsymbol{y}$ such that $\dot{F}(\boldsymbol{y})=1$, a compact set. Hence, for each subset $S, F_{m}(\overline{\boldsymbol{y}}) / F_{m}(\boldsymbol{y})$ has a maximum $e_{S}$. Set $e=\max e_{S}$.

Lemma 2. Let $\Omega$ be the set of solutions of (1), let $\boldsymbol{x}$ be a point exterior to $\Omega$, and let $\boldsymbol{y}$ be the point in $\Omega$ nearest to $\boldsymbol{x}$. Let $S$ be the subset of the half spaces (1), each of which contains $y$ in its bounding hyperplane, and let $\Omega_{S}$ be the intersection of these half spaces.

Then $\boldsymbol{x}$ is exterior to $\Omega_{S}$ and $\boldsymbol{y}$ is the nearest point of $\Omega_{S}$ to $\boldsymbol{x}$.

Lemma 3. Let $M$ be an $m \times n$ matrix obtained from $A$ by substituting 0 for some of the rows of $A$, and let $\Omega$ be the cone of solutions of $M z \leqq 0$. Let $E$ be the set of all points $x$ such that (i) $\boldsymbol{x}$ is exterior to $\Omega$, and (ii) the origin is the point of $\Omega$ nearest to $\boldsymbol{x}$.

Then there exists a $d_{S}>0$ such that $\boldsymbol{x} \in E$ implies

$$
F_{m}\left((M \boldsymbol{x})^{+}\right) \geqq d_{S} F_{n}(\boldsymbol{x})
$$

Proof of the theorem. Let $\boldsymbol{x}$ be any vector exterior to the solutions $\Omega$ of (1), let $\boldsymbol{x}_{0}$ be the point of $\Omega$ nearest to $\boldsymbol{x}$, and let $S$ be defined as in lemma 2 .

Let $M$ be the matrix obtained from $A$ by substitut-

2 S. Agmon, The relaxation method for linear inequalities, National Applied Mathematics Laboratory Report 52-27, NBS (prepublication copy). 
ing 0 for the rows not in $S$, and let $\overline{\boldsymbol{b}}$ be the vector obtained from $\boldsymbol{b}$ by substituting 0 for the components not contained in $S$.

Then lemma 2 says that $\boldsymbol{x}_{0}$ satisfies $M \boldsymbol{z}=\overline{\boldsymbol{b}}, \boldsymbol{x}$ is exterior to the solutions of $\boldsymbol{M z} \leqq \overline{\boldsymbol{b}}$, and $\boldsymbol{x}_{0}$ is the solution of $M z \leqq \overline{\boldsymbol{b}}$ nearest to $\boldsymbol{x}$. Perform the translation $\boldsymbol{z}^{\prime}=\boldsymbol{z}-\boldsymbol{x}_{0}$. Then $M \boldsymbol{z} \leqq \overline{\boldsymbol{b}}$ if, and only if,

$$
M z^{\prime}=M z-M x_{0}=M z-\bar{b} \leqq 0 .
$$

Thus $\boldsymbol{x}-\boldsymbol{x}_{0}$ belongs to the set $E$ of lemma 3 , and

$$
\begin{gathered}
F_{m}\left((M \boldsymbol{x}-\overline{\boldsymbol{b}})^{+}\right)=F_{m}\left(\left(M\left(\boldsymbol{x}-\boldsymbol{x}_{0}\right)\right)^{+}\right) \geqq d_{S} F_{n}\left(\boldsymbol{x}-\boldsymbol{x}_{0}\right) \geqq \\
d F_{n}\left(\boldsymbol{x}-\boldsymbol{x}_{0}\right),
\end{gathered}
$$

where $d=\min _{S} d_{S}$.

Thus,

$$
F_{n}\left(\boldsymbol{x}-\boldsymbol{x}_{0}\right) \leqq \frac{1}{d} F_{m}(M \boldsymbol{x}-\overline{\boldsymbol{b}}) \leqq \frac{e}{d} F_{m}\left((A \boldsymbol{x}-\boldsymbol{b})^{+}\right),
$$

using lemma 1. Setting $c=e / d$ completes the proof of the theorem.

\section{Estimates of $c$ for various norms}

None of the estimates to be obtained is satisfactory, since each requires an inordinate amount of computation except in special cases. It is worth remarking, however, that even without knowledge of the size of $c$, the theorem is of use in insuring that any computation scheme that makes $(A \boldsymbol{x}-\boldsymbol{b})^{+}$ approach 0 will bring $x$ close to the set of solutions of (1). This guarantees, for instance, that in Brown's method for solving games the computed strategy vector is approaching the set of optimal strategy vectors.

In what follows let

$\boldsymbol{x}=$ maximum of the absolute values of the coordinates of $\boldsymbol{x}$;

|| $\boldsymbol{x}||=$ sum of the absolute values of the coordinates of $\boldsymbol{x}$;

||$|\boldsymbol{x}|||=$ the square root of the sum of the squares of the coordinates of $\boldsymbol{x}$.

Note that if $F_{m}$ is any one of these norms, then $e=1$. We consider these cases:

Case I. $\quad F_{n}=\|||||, F_{m}=|\quad|$. If $C=\left(c_{i j}\right)$ is a square matrix of $r$ th order, let

$$
\Gamma(C)=\left[\sum_{j=1}^{r}\left(\sum_{i=1}^{r}\left|C_{i j}\right|\right)^{2}\right]^{\frac{1}{2}},
$$

where the $C_{i j}$ 's are the cofactors of the elements of $c_{i j}$. Using this notation, and assuming that the rows of (1) are normalized so that $\sum_{j=1}^{n} a_{i j}{ }^{2}=1$, Agmon (see p. 9 of reference in footnote 2 ) has shown that if
$A$ is of rank $r$, then

$$
C \leqq \frac{\left[{ }_{1 \leq j_{1}<\ldots<j_{r} \leq n}\left(\Gamma_{i_{1}, \ldots, i_{r_{r}}}^{j_{1}, \ldots}\right]^{2}\right]^{\frac{1}{2}}}{\left[\sum_{1 \leq j_{1}<\ldots<j_{r} \leq n}\left|A_{i_{1}, \ldots, j_{i_{r}}}^{j_{1}}\right|^{2}\right]^{\frac{1}{2}}},
$$

where $i_{1}, \ldots, i_{\mathrm{r}}$ are $r$ (fixed) linearly independent rows of $\left(a_{i j}\right) ; A_{i_{1}, \ldots, i_{i_{r}}}^{j_{1}, \ldots}$ is the $r \times r$ submatrix formed by the fixed rows and indicated columns, and where the summation is performed over all different combinations of the $j$ 's.

Case II. $\quad F_{n}=|\quad|, F_{m}=|\quad|$.

Case III. $\quad F_{n}=|\quad|, F_{m}=\|\quad\|$.

For cases II and III, it is convenient to have a description of $E$ alternative to that contained in lemma 3. We shall use the notation of lemma 3.

Lemma 4. Let $K^{\prime}=$ the cone spanned by the row vectors of $M$, with the origin deleted. Then $K^{\prime}=E$.

Proof. Let $M_{1}, \ldots, M_{m}$ be the row vectors of $M$, and let $\boldsymbol{x}=\lambda_{1} M_{1}+\ldots+\lambda_{m} M_{m}$, where $\boldsymbol{x} \neq \mathbf{0}$, and $\lambda_{i} \geqq 0, i=1, \ldots, m$. Then $\boldsymbol{x}$ is exterior to $\Omega$, and the origin is the point of $\Omega$ closest to $x$; that is, $z \in \Omega$ implies $(\boldsymbol{x}-\boldsymbol{z}) \cdot(\boldsymbol{x}-\boldsymbol{z})-\boldsymbol{x} \cdot \boldsymbol{x} \geqq 0$. For $\boldsymbol{z} \cdot \boldsymbol{x}=\boldsymbol{z} \cdot\left(\lambda_{1} M_{1}+\right.$ $\left.\ldots+\lambda_{m} M_{m}\right)=\lambda_{1} z \cdot M_{1}+\ldots+\lambda_{m} z \cdot M_{m} \leqq 0$. Hence $(\boldsymbol{x}-\boldsymbol{z}) \cdot(\boldsymbol{x}-\boldsymbol{z})-\boldsymbol{x} \cdot \boldsymbol{x}=\boldsymbol{z} \cdot \boldsymbol{z}-2 \boldsymbol{z} \cdot \boldsymbol{x} \geqq 0$. This shows that $K^{\prime} \subset E$.

We now prove that $E \subset K^{\prime}$. Assume $\boldsymbol{x} \in E$. Then,

$$
z \epsilon \Omega \text { implies } z \cdot x \leqq 0
$$

(otherwise let $w$ be a sufficiently small positive number; then $w \boldsymbol{z} \in \Omega$ and $w \boldsymbol{z} \cdot w \boldsymbol{z}-2 w \boldsymbol{z} \cdot \boldsymbol{x}<0)$. Consider $z$ as the coordinates of a half space whose bounding plane contains the origin. Then (4) says that all half spaces ("through" the origin) containing the row vectors of $M$ also contain $\boldsymbol{x}$. It is a fundamental result in the theory of linear inequalities ${ }^{3}$ that this later statement implies that $\boldsymbol{x}$ is in the cone generated by the rows of $M$. Hence $E \subset \mathrm{K}^{\prime}$.

\section{Case II}

It is clear from the proof of the theorem that all we need is to calculate $\left.\min _{x \in E}(\mid \boldsymbol{M} \boldsymbol{x})^{+}|/| \boldsymbol{x} \mid\right)$, for each $M$ corresponding to a subset $S$ of the vectors $A_{1}$, . . ., $A_{m}$. Let $A_{1}, \ldots, A_{k}$ (say) be the vectors of the subset $S$. Then by lemma $4, x \in E$ implies that there exist $\lambda_{1}, \ldots, \lambda_{k}$ with $\lambda_{j} \geqq 0$ such that

Hence,

$$
\boldsymbol{x}=\lambda_{1} A_{1} \neq \ldots+\lambda_{k} A_{k} .
$$

$$
|\boldsymbol{x}| \leqq a_{S} \sum_{j=1}^{k} \lambda_{j}
$$

where $a_{S}$ is the largest absolute value of the co-ordinates of $A_{1}, \ldots, A_{k}$.

It follows from the homogeneity of $\left|(M \boldsymbol{x})^{+}\right| /|\boldsymbol{x}|$ that

3 T. S. Motzkin, Beiträge zur Theorie der Linearen Ungleichungen. Jerusalem, 1936, with references to proofs by Minkowski and Weyl. 
we need only consider $\boldsymbol{x} \epsilon E$ such that if $x$ is expressed as in $(5), \sum_{j=1}^{k} \lambda_{j}=1$.

Then

$$
\begin{aligned}
\left|(\boldsymbol{M} \boldsymbol{x})^{+}\right|=\max _{i}\left(A_{i} \cdot \boldsymbol{x}\right)^{+}=\max _{i}\left(A_{i} \cdot \boldsymbol{x}\right) & = \\
\max _{i}\left(A_{i} \cdot \sum_{j=1}^{k} \lambda_{j} A_{j}\right) & =\max _{i} \sum_{j=1}^{k} g_{i j} \lambda_{j},
\end{aligned}
$$

where $g_{i j}=A_{i} \cdot A_{j}, \lambda_{j} \geqq 0, \sum \lambda_{j}=1$.

Hence,

$$
\min \left|(M \boldsymbol{x})^{+}\right|=\min _{\lambda} \max _{i} \sum_{j=1}^{k} g_{i j} \lambda_{j}=v_{S},
$$

where $v_{S}$ is the value of zero sum two person game whose matrix is $g_{i j}$.

Therefore, from (6) and (7)

$$
\min _{x \in E} \frac{\left|(M \boldsymbol{x})^{+}\right|}{|\boldsymbol{x}|} \geqq \frac{v_{S}}{a_{S}} .
$$

Can $v_{s}=0$ ? Clearly, if, and only if, the origin is in convex body spanned by $A_{1}, \ldots, A_{k}$. But this would imply that the set $E$ is the entire space (except for the origin). And it follows from the proof of the main theorem that this can occur only for a subset $S$ that would never arise in lemma 2.

Therefore, using the language of the theorem

where

$$
\left|\boldsymbol{x}-\boldsymbol{x}_{0}\right| \leqq c\left|(A \boldsymbol{x}-\boldsymbol{b})^{+}\right|,
$$

$$
c=\max _{v_{\mathrm{S}}>0} \frac{a_{S}}{v_{S}} .
$$

A special case occurs when all $A_{i} \cdot A_{j}>0$. Let $v=\min _{i, j} A_{i} \cdot A_{j}, a=\max _{i, j}\left|a_{i j}\right| . \quad$ Then,

$$
\left|\boldsymbol{x}-\boldsymbol{x}_{0}\right| \leqq \frac{a}{v}\left|(A \boldsymbol{x}-\boldsymbol{b})^{+}\right| .
$$

\section{Case III}

Reasoning, along the lines of case II, we need only estimate $\min _{\lambda}\left\|\left(\sum_{j=1}^{k} g_{i j} \lambda_{j}\right)^{+}\right\|$, and it is possible to derive from it an expression analogous to (8), which unfortunately does not seem to have a neat statement in terms of games or any other familiar object. An interesting special case occurs, however, if the matrix $g_{i j}$ (for $S$ all the rows of A), has the property that

$$
w=\min _{i}\left(g_{i i}+\sum_{\substack{j=1 \\ g_{i j}<0}}^{n} g_{i j}\right)>0 .
$$

Then

$$
\begin{aligned}
\min \left\|\left(\sum_{j=1}^{k} g_{i j} \lambda_{j}\right)^{+}\right\| & \geqq \min _{\lambda} \sum_{i=1}^{k} \sum_{j=1}^{k} g_{i j} \lambda_{j} \\
& =\min _{\lambda} \sum_{j=1}^{k} \lambda_{j} \sum_{i=1}^{k} g_{i j} \geqq \min _{\lambda} \sum_{j=1}^{k} \lambda_{j} w=w .
\end{aligned}
$$

Then we obtain, with $a$ having the same meaning as in (9)

$$
\left|\boldsymbol{x}-\boldsymbol{x}_{0}\right| \leqq \frac{a}{w}\left\|(A \boldsymbol{x}-\boldsymbol{b})^{+}\right\| .
$$

Washington, June 5, 1952. 